\title{
Interventional radiology and COVID-19: How to face the challenge?
}

\author{
Sreedhara B Chaluvashetty, Naveen Kalra, Harish Bhujade, Shyamkumar N Keshava', Chander Mohan² \\ Department of Radiodiagnosis and Imaging, Postgraduate Institute of Medical Education and Research, Chandigarh, ${ }^{1}$ Department \\ of Radiology, Christian Medical College, Vellore, Tamil Nadu, ${ }^{2}$ Department of Interventional Radiology, BLK Superspeciality \\ Hospital, New Delhi, India \\ Dr Naveen Kalra is a Member of the Interventional Radiology Subspecialty Group of IRIA/ICRI and Dr Chander Mohan is the Head \\ of the Interventional Radiology Subspecialty group of IRIA/ICRI
}

Correspondence: Dr. Naveen Kalra, Department of Radiodiagnosis and Imaging, Nehru Hospital, Postgraduate Institute of Medical Education and Research, Chandigarh-160 012, India. E-mail: navkal2004@yahoo.com

\begin{abstract}
With the sudden outbreak of Coronavirus disease-19 (COVID-19) in China, and its rapid spread across the continents over a short period of time, healthcare workers are posed with the challenge of managing these patients as well protecting themselves from getting infected. Since interventional radiology deals with both elective and emergency services, wherein close patient contact is a norm, there is a substantial risk of acquiring and transmitting infection. Given the circumstances, it is imperative to develop broadly applicable guidelines to utilize the available resources in an optimal fashion and limit transmission of disease. This brief review deals with infection control measures within the Interventional Radiology department or section and possible recommendations that can be adopted at the institutional level.
\end{abstract}

Key words: COVID-19; interventional radiology; protocol and guidelines

\section{Introduction}

Since the onset of global Coronavirus Disease 2019(COVID-19) pandemic in December 2019 in Wuhan, China, there has been a rapid and exponential increase in the active infection as well as mortality all over the world. In India, there is gradual to rapid increase in the number of positive cases since March last week with more than 200000 cases reported at the time of this article ${ }^{[1]}$ With the variability in asymptomatic phase of infection and asymptomatic carriers acting as a source of infection spread, containment of disease spread along with treatment has become the major goal for the government as well as healthcare providers. Like with other surgical and medical departments, Interventional Radiology (IR) section should also continue its services safely and effectively while taking necessary precautions in reducing the risk of

\begin{tabular}{|l|l|}
\hline \multicolumn{2}{|c|}{ Access this article online } \\
\hline Quick Response Code: & \\
\cline { 1 - 2 } & Website: \\
\hline & www.ijri.org \\
\cline { 2 - 3 } & DOI: \\
\cline { 1 - 2 } & 10.4103/ijri.IJRI_351_20 \\
\hline
\end{tabular}

transmission to the staff involved in procedures as well as other patients seeking the services within the IR section. With the so far data available about COVID-19 and its mode of transmission, IR needs to make satisfactory and acceptable protocols and guidelines that maintain optimal patient care without compromising on precautionary measures for the IR staff.

\section{Workforce Segregation and Functioning of IR Section}

In the context of the rapid increase in COVID positive patients over the last few days, the healthcare system in

This is an open access journal, and articles are distributed under the terms of the Creative Commons Attribution-NonCommercial-ShareAlike 4.0 License, which allows others to remix, tweak, and build upon the work non-commercially, as long as appropriate credit is given and the new creations are licensed under the identical terms.

For reprints contact: WKHLRPMedknow_reprints@wolterskluwer.com

Cite this article as: Chaluvashetty SB, Kalra N, Bhujade $\mathrm{H}$, Keshava SN Mohan C. Interventional radiology and COVID-19: How to face the challenge? Indian J Radiol Imaging 2021;31:S38-44.

Received: 09-May-2020 Revised: 29-May-2020

Accepted: 06-Jul-2020 Published: 23-Jan-2021 
India is expected to function at maximum capacity for many months. Hence, it is important to prioritize resources like ventilators, personal protective equipment (PPE), and healthcare personnel. Unlike PPE or ventilators, healthcare workers cannot be "manufactured" in short duration of time or run at full occupancy for longer periods. In addition, there is always a risk of staff coming in contact with a COVID-19 patient, who in turn risk exposing multiple other healthcare workers to the contagion. It is important to reduce the overall workload of the IR department and segregate the workforce which can be physical or temporal. Temporal segregation requires dividing IR staff into 2 or 3 teams and working at reduced strength while the other team(s) stay at home.

This may not be a feasible option in many setups due to limited IR staff and, hence, physical segregation appears to be a more plausible option. Each team should include interventional radiologist, nursing staff, technician, and other support staff, who are necessary for complete patient care and have the capability to provide the full spectrum of the IR service.

Complete vertical segregation of the workforce should be maintained at all levels so that care can be provided without interruption in case a member of the alternating team needs to go into isolation. It is also recommended that these units refrain from contact with each other. ${ }^{[2,3]}$

\section{Vetting and Prioritization of IR Procedures}

Since there is considerable risk of acquiring infection through the asymptomatic cases or asymptomatic carriers, to reduce exposure and workload, IR procedures should be predominantly limited to emergency cases with postponement or spacing of elective cases till such time the situation is brought under control.

\section{Emergency Interventions}

The IR-related emergencies that require immediate intervention are acute ischemic stroke, acutely ruptured aneurysms, life-threatening hemorrhages (traumatic and inflammatory/infective etiology), vascular complications of acute pancreatitis, critical limb ischemia, acute symptomatic iliofemoral deep vein thrombosis (DVT), acute mesenteric ischemia, symptomatic or abdominal aortic aneurysm greater than $5.4 \mathrm{~cm}$, and massive pulmonary embolism in non-COVID patients. ${ }^{[4]}$ Nonvascular emergencies like interventional pain management for severe acute pain or severe exacerbation of chronic underlying condition, kyphoplasty/vertebroplasty for compression fractures with severe acute pain, percutaneous drainages for large collections and abscesses, percutaneous transhepatic biliary drainage, and percutaneous nephrostomies also require immediate attention. Table 1 highlights the examples of emergency IR procedures; however, the list need not be restricted to what has been mentioned. Whenever there is a doubt, case by case approach is needed. In such situations, interdepartmental discussions will be useful in arriving a suitable decision. Oncological procedures like transarterial chemoembolization, transarterial radioembolization, tumor ablations, biopsies, and port insertions should be spaced evenly to reduce workload. Few of the semiurgent procedures would require an assessment at the individual level for disease morbidity and mortality before any decision is taken [Table 1].

\section{Elective Procedures}

The nonurgent elective procedures that can be postponed for 1 to 3 months include fine needle aspiration cytology for benign entities like thyroid nodules and granulomas, peripheral arteriovenous malformation embolization, prostate artery embolization, fibroid embolization, varicocele embolization, angioplasty for Rutherford 1-3 (claudication), asymptomatic visceral artery or carotid artery stenosis, asymptomatic DVT, asymptomatic abdominal aortic aneurysms less than $5.4 \mathrm{~cm}$, and CEAP class $1-5$ chronic venous insufficiency [Table 1$] \cdot{ }^{[4]}$ All those patients who have been scheduled for elective procedures should be contacted and informed about the decision and counselled about the future course of action depending on the healthcare crisis.

\section{IR in Suspected or Positive COVID Patients}

Modes of transmission, precautions, and personal protective equipment

The standard protocol of infection control and prevention is increasingly relevant in the present scenario. Standard precautions apply to all patients at all times irrespective of their infective status and are the mainstay of infection control. Personal protective equipment is the protective equipment worn to prevent exposure to hazardous biological or chemical agents. Based on the mode of disease transmission, they can be simple, such as face masks and nonsterile examination gloves, or complex, such as positive-pressure isolation suits worn in high-containment laboratories..$^{[5]}$

Surgical masks are fluid resistant and primarily protect from splashes of body fluids and blood, reducing droplet transmission. Respirators are protective equipment that filters out airborne particles, as well as toxic gases and vapors. The N95 respirators filter out $95 \%$ of airborne particles of particulate size less than $0.3 \mu \mathrm{m}$, and are the USA equivalent of the European FFP2/FFP3 (filtering facepieces) masks which filter at least $94 \% / 99 \%$ of particles, respectively. Alternatively, power air-purifying respirators which contains half-face mask, helmet/hood or 
Chaluvashetty, et al.: Challenges for interventional radiology in the times of COVID-19

Table 1: Broader classification of IR services for the management of resources and reduction of cross-transmission

\begin{tabular}{ll}
\hline Emergency IR services & Elective services \\
\hline Vascular Procedures & Peripheral AVM embolization \\
Acute ischemic stroke & Prostate artery embolization \\
Acutely ruptured aneurysms & Fibroid embolization \\
Life-threatening hemorrhages & Varicocele embolization \\
Vascular complications of acute pancreatitis & Angioplasty for Rutherford 1-3 claudication \\
Critical limb ischemia & Asymptomatic visceral artery or carotid artery \\
Acute symptomatic iliofemoral deep vein thrombosis & stenosis \\
Acute mesenteric ischemia & Asymptomatic deep vein thrombosis \\
Symptomatic abdominal aortic aneurysm $>5.4 \mathrm{~cm}$ & Asymptomatic adominal aortic aneurysms $<5.4$ mm \\
Massive pulmonary embolism & CEAP class 1-5 chronic venous insufficiency \\
Port placement/vascular access & FNAC/Biopsy of suspected benign etiologies like \\
Nonvascular Interventions & thyroid nodules \\
Interventional pain management for & \\
severe acute pain & \\
Kyphoplasty/Nertebroplasty for compression & \\
fractures with severe acute pain & \\
Percutaneous drainages for a large collection and abscess & \\
Percutaneous transhepatic biliary drainage and percutaneous & \\
nephrostomies in acutely infected system & \\
Procedures that are semiurgent and can be spaced at individual discretion & \\
based on the stage of presentation & \\
Transarterial Chemoembolization (TACE) & \\
Transarterial Radioembolization (TARE) & \\
Ablations of tumors & \\
Biopsies of suspected malignant lesions & \\
\hline
\end{tabular}

full facepiece attached to a battery pack that forces room air through a filter can be used by individuals who cannot wear a particulate respirator due to comorbidities or difficulty in breathing for aerosol-generating procedures. ${ }^{[2,5]}$ Eye protection devices include goggles or safety glasses and face shield which protect the eye from splashes of body fluids or chemicals.

According to the available data, severe acute respiratory syndrome Coronavirus 2 (SARS-CoV-2) transmission is primarily through droplet transmission and contact routes (fomite transmission) with chances of airborne transmission in specific circumstances like aerosol-generating procedures. ${ }^{[6]}$ In the context of varying incubation period and asymptomatic carriers, the N95 respirators or FFP2 masks and eyeshield are must for all interventions irrespective of their COVID status, whereas aerosol-generating procedures like pleural drainage, lung biopsies, lung ablations, and bronchial artery embolization require face shield for extra protection [Table 2]. For COVID positive patients, complete set of PPE includes two sets of gloves, full length long-sleeved impervious gowns that cover entire body surface, shoe covers, N95/FFP2 masks, eye protection (face shield or goggles), surgical caps, and face cover. ${ }^{[2]}$ Complete PPE is a must for all the staff involved in patient shifting, preparation, nursing staff, technical assistants, residents, and faculty.

\section{Staff protection measures}

Since IR staff are involved in examining, interacting, and treating patients directly, they are at risk of a high degree of infection transmission. COVID being a respiratory infection
Table 2: Aerosol-generating procedures performed in the IR suite

Lung biopsy
Pleural fluid aspiration and drainage
Chest tube for pneumothorax
Lung tumor ablation
Bronchial artery embolization
Percutaneous/endovascular embolization of Rasmussen aneurysm
Bronchial stenting
Nasogastric tube (NG tube) placement
Gastrostomy and Gastro-jejunostomy tube placement
Gastrointestinal stent placement

with droplet and contact transmission, it is imperative to have handwashing and infection control measures in place. All the staff members (faculty, technologists, nursing staff and hospital attendants) should have received adequate training from the hospital and department COVID team for maintaining personal and hand hygiene during and after the procedure, when and what PPE to use, how to don and doff PPE to prevent self-contamination, and how to dispose of PPE.

Hand hygiene should be performed with alcohol-based hand rub containing at least $60 \%-95 \%$ alcohol or by washing hands with soap and water for at least $20 \mathrm{~s}$ as described in Figure 1. It should be done before and after all patient interaction or contact with potentially infectious material, and before putting on and after removing PPE. The sequence of putting on and removing PPE is demonstrated in Figures 2 and 3 and is also available online. ${ }^{[7,8]}$ Healthcare personnel should be able to demonstrate competency in performing appropriate infection control practices and procedures before caring for patients. Respirator or facemask should be donned before entry into the patient area. Disposable 
respirators and facemasks should be discarded after exiting the patient's care area and closing the door. ${ }^{[9]}$

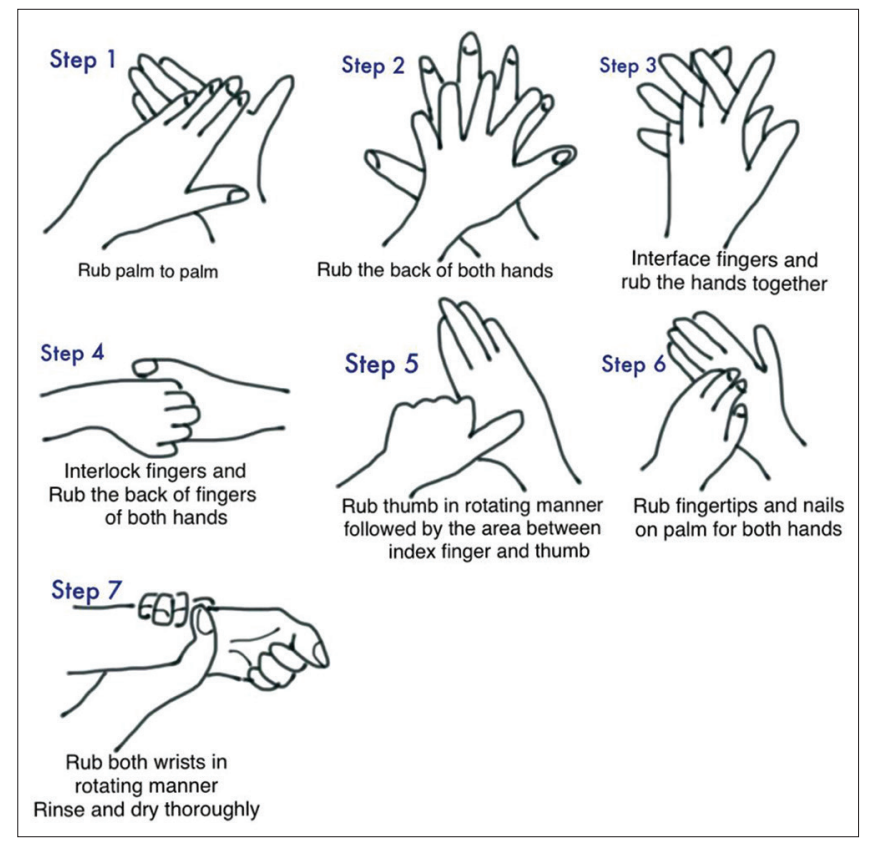

Figure 1: Hand wash technique

\section{Functional area or procedure room}

To simplify the functioning of IR, the procedures and functional area should be ideally segregated into COVID and non-COVID patients, and all suspected COVID patients should be tested for COVID before considering for any interventions.

All ultrasound-guided procedures for COVID positive patients should be done at the bedside. For fluoroscopic-guided procedures, the patient needs to be shifted to the IR suite. Institutes or hospitals with 2 or more IR suites should earmark 1 IR suite exclusively for COVID positive patients to reduce the chance of cross-transmission.

In a setup with single IR suite wherein complete isolation from rest of the department is not feasible, the interventional area must be separated from the rest of the department with a controlled environment and access, compatible with an ISO 7 level of cleanroom class determined by acceptable maximum concentration limit of particles of size $\geq 0.5 \mu \mathrm{m}$ and above to be less than 352000, and on an average of 30-60 air changes per hour through the HEPA air filters within the IR suite/cleanroom. ${ }^{[9,10]}$ The interventions for COVID positive patients should be done preferably as

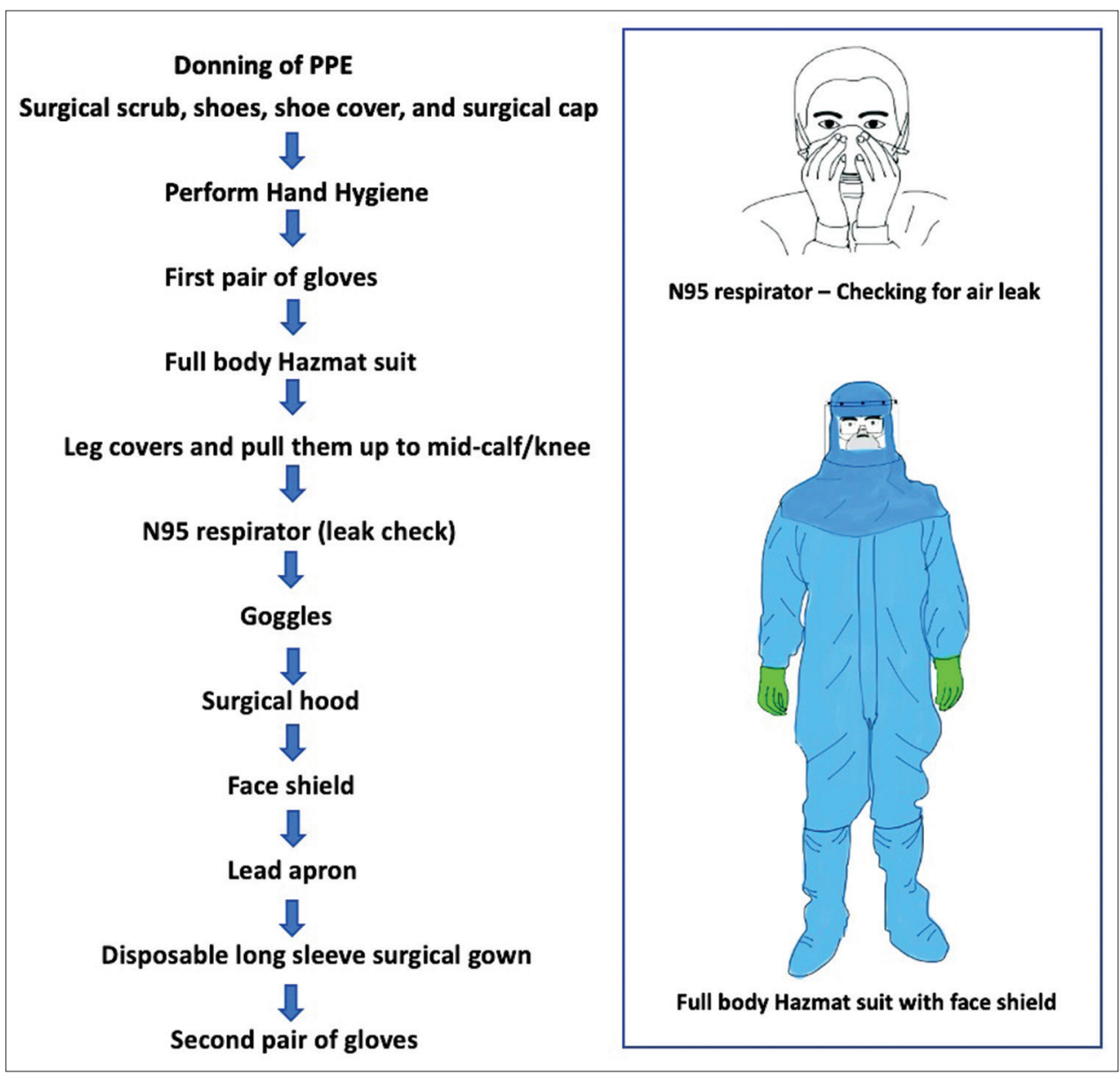

Figure 2: Steps of donning of PPE in IR suite 
the last case of the day with necessary precautions and post-procedure disinfection and fogging of the DSA suite to reduce cross-transmission. If the procedure is done earlier in the day, the room should be kept free after cleaning for approximately more than 1 hour. A similar practice is to be followed for CT-guided procedures also.

There must be separate areas/rooms for donning and doffing. Both areas should have full-length mirrors, lead hangers, and hand sanitizer. The flow chart of steps of donning and doffing should be kept in respective areas. Doffing room should have a separate marking of clean and dirty areas with two labelled containers (yellow color bag for disposable items and red color bag for reusable items) to be placed in the dirty area.

Biomedical waste (BMW) should be collected at one specified place within the lab in a container, which will be disposed of by the support staff wearing full PPE. BMW should be disposed of as per hospital biomedical management guidelines. Specific importance should also be given to developing a patient shifting corridor from the ward/ICU to the IR suite.

\section{Patient requisition, consent, and preparation}

All suspected patients should have had COVID testing according to the ICMR/Hospital guidelines before entering the IR department. The clinical teams should be clearly directed to let the IR team know when a procedure is being requested for a confirmed COVID-19 patient. Requisition form should not be accepted physically from the clinical team, instead, the form would be prepared by the IR staff after verifying from the file where the procedure requested should be clearly mentioned. Specific care should also be taken while dealing with patient radiology and documents. A view box and desk should be specifically earmarked for the same, which should be cleaned with alcohol disinfectant

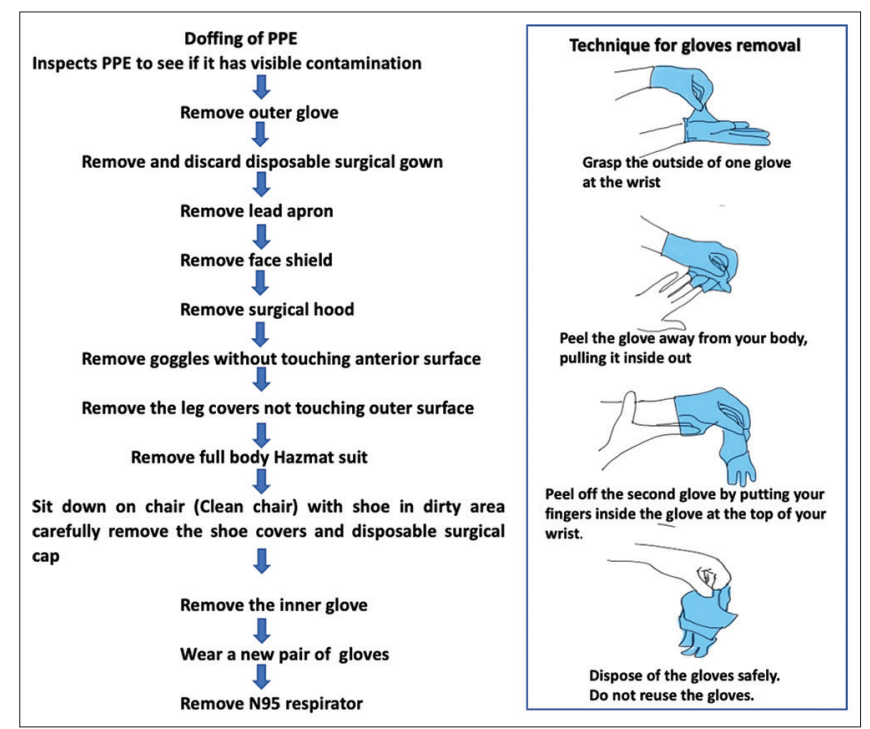

Figure 3: Steps of doffing of PPE after returning the documents. All patients should be wearing N95 or equivalent masks, surgical caps, and gown before entering the IR premises. The patients' attendants and hospital staff involved in patient shifting should be wearing N95 or equivalent masks and eye protection/face shield. Patient movement should be restricted to designated area, and laboratory/biochemical workup and portable imaging should be performed at the bedside whenever feasible.

Following the patient transfer, entry of all personnel into the procedure room should be allowed only after sufficient time has elapsed for air changes to remove potentially infectious particles. A simplified workflow chart [Figure 4] can be prepared based on the hospital set up for smooth functioning of the section and to protect health care workers and patients from acquiring an infection while at the same time providing IR services to the patients who deserve it.

\section{Cleaning and Disinfection}

The IR department should develop a policy to clean, sanitize, and disinfect all the environmental surfaces exposed during interventions. General principles of cleaning and sanitation should be maintained regardless of the COVID positivity. Neutral detergent and warm water solution should be used for all routine and general cleaning purposes.

To disinfect the contaminated area with spillage of blood or body fluids, $1 \%$ sodium hypochlorite solution is recommended. The spill should be covered with $1 \%$ sodium hypochlorite solution for 10-20 min followed by cleaning up of the spill and discard into the infectious waste bin. Subsequently cleaning of the area with a detergent solution and warm water is recommended, especially to protect metal surfaces from corrosive effects of bleaching solution or

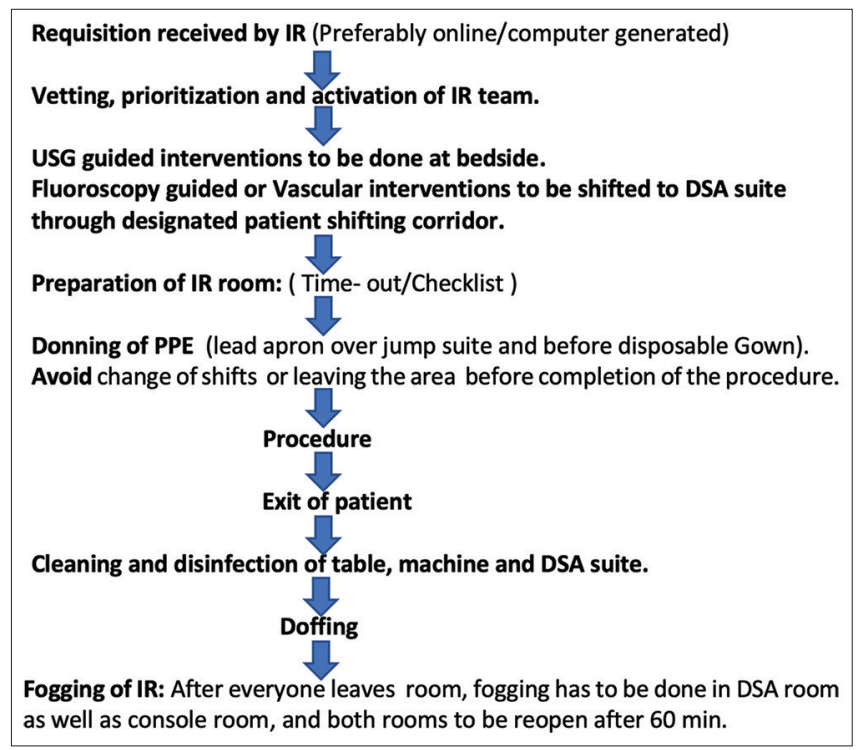

Figure 4: Simplified flow chart for IR services 
powder. For large quantity spills, it is advisable to use $10 \%$ sodium hypochlorite as a disinfectant. Since bleach solution becomes rapidly unstable, daily fresh preparation or changing on becoming dirty/turbid is suggested. For smooth metal surfaces, tabletops and other surfaces on which bleach cannot be used, alcohol (70\% and above), isopropyl, ethyl alcohol, or methylated spirit should be used. ${ }^{[11]}$

While using ultrasound during IR procedures, it is advisable to use probe covers and transparent drapes over the ultrasound system to reduce the contamination from direct patient contact and droplets transmission. Once the patient is shifted, these covers should be discarded and the ultrasound system including keyboard, screen, handlebar, cable, and transducer should be initially cleaned with a mild soft cloth moistened with mild detergent/soap water, followed by water. For chemical disinfection, low-level disinfectant like sodium hypochlorite, $60 \%-70 \%$ ethanol, $0.5 \%$ hydrogen peroxide, or $70 \%$ isopropyl alcohol can be used, and preferably by using a soaked gauze or cloth to wipe the surfaces rather than direct pouring on these surfaces. The instructions and recommendation for cleaning the particular ultrasound system during COVID-19 are also available at manufacture's website. Similar cleaning and disinfection protocol is followed to disinfect CT gantry as well. ${ }^{[11-14]}$

Ultraviolet (UV) disinfecting devices use UVA or UVC light to produce a germicidal effect. UV light is capable of destroying viruses, bacteria, and fungi. The SARS-CoV-2 virus has not yet been specifically tested for its ultraviolet susceptibility; however, many other tests on related coronaviruses have concluded that they are highly susceptible to ultraviolet inactivation. They are intended to augment disinfection of health care environmental surfaces after manual cleaning has been performed. These UV disinfecting devices can also be used to sterilize reusable protective equipment like face shield and respirators/ masks. ${ }^{[15]}$

All work stations, IR suites, and departmental areas in use should be cleaned and disinfected before and after a new team member uses it. Apart from this, mandatory cleaning should be undertaken at least 3-4 times a day to reduce any risk of transmission.

\section{IR in Non-COVID Patients}

All inpatient and emergency IR services continue to operate for non-COVID patients in the routine designated area within the IR section with specific precautions to avoid transmission from asymptomatic cases or carriers. All patients coming to IR should be screened with a standard questionnaire and temperature check for COVID status at the nursing station before interventions, irrespective of clinical status and procedure requested. In case of any suspicion, rapid isolation of the patient, the immediate contact of hospital infection control committee, and testing for SARS CoV2 should be contemplated.

All patients and attendants visiting the department should be wearing surgical masks compulsorily. Not more than one visitor should be allowed to accompany the patient, and the visitor should also be assessed regarding travel history and clinical symptoms. If found to have affirmative history, the visitor should be asked to leave the premises.

Staff protection measures: Surgical mask is advised in dealing with low-risk patients without any risk factors for COVID-19 disease. For patients with moderate to high risks like patients with pneumonia and those who are under quarantine for close contact with COVID-19 patients, N95/ FFP2 mask is recommended. Some of the aerosol-generating procedures (AGP) in IR include pleural drainage, lung biopsy (chances of hemoptysis during the procedure which can incite violent coughing and aerosolization), ablation of lung tumors, and bronchial artery embolization. For these procedures, eye protection with N95/FFP2 mask is recommended. ${ }^{[2]}$

\section{Staff Surveillance and Social Distancing}

Temperature monitoring of all staff is recommended at least twice daily. This surveillance helps to identify fever clusters among the IR staff which could indicate disease transmission among HCWs. Staff who are unwell should be given leave and monitored appropriately.

Many reports have shown that people with comorbidities like age above 60 years, smokers, hypertensives, diabetics, chronic kidney disease, chronic heart disease, those with previous lung damage, and cancer are at higher risk of acute respiratory distress syndrome if infected. Exclusion of these IR staff from coming into contact with confirmed COVID-19 cases is prudent. ${ }^{[9]}$

Social distancing is one of the key strategies to prevent HCW to HCW infection. Hence, all referrals to IR should be by phone or by online request avoiding face-to-face consult as much as possible. Video or teleconferencing should be used for meetings, including multi-disciplinary team discussions and tumor board. HCWs are most vulnerable for infection transmission during lunchtime as masks are removed. Hence, all staff should be encouraged to have their lunch individually or in small groups within their respective teams, maintain a safe distance between them. With community spread becoming more rampant over a while, the chances of health care worker acquiring COVID infection within the community may be higher than at the workplace. If social distancing measures are not implemented at the institutional and community level, a large group of HCW can potentially be infected or quarantined if any of the staff 
members acquire COVID-19. ${ }^{[2]}$ At present, there is no strong recommendation for prophylactic drugs.

\section{Real life challenges}

There are patients with medical and surgical emergencies with asymptomatic COVID positive status requiring emergency interventions like stroke or life-threatening bleeding. In the initial days, we had to perform interventions without knowing COVID status due to limited availability of the testing kits and time required for testing. In such circumstances, we took complete protection using PPE kits with proper donning and doffing, and we did a case of bronchial artery embolization for massive hemoptysis and acute ischemic stroke intervention in one patient without knowing the COVID status. Another common challenge that we encountered was with respect to diagnostic procedures like biopsies and drainages wherein it was impossible to get COVID status checked for all patients due to large volume of cases, and hence we adopted to doing all procedures with N95 mask, face shield, and impervious gowns for self-protection.

Considering the dynamic nature of the COVID pandemic, it is very important to keep up to date with regards to the disease spread, IR society recommendations, as well as government regulations. Some journals have published rapid research and review articles. ${ }^{[9,16,17]}$ Regular meetings and trainings are important at the hospital/institutional levels.

\section{Conclusion}

With a rapid increase in the number of COVID positive patients, it is important to understand the disease, symptoms/presentation, and mode of transmission so that necessary precautions and measures can be taken to protect healthcare workers and prevent cross-transmission while delivering the required IR services. The above recommendations can be used as a framework on which specific protocol can be set at the institutional level to reduce risk to staff and deliver quality patient care. The IR section should continue the service especially for emergency procedures and important elective procedures in these unprecedented times. As the knowledge about COVID-19 infection keeps increasing, the guidelines and recommendations for infection control for this pandemic may also keep changing making it a dynamic process.

\section{Financial support and sponsorship}

Nil.

\section{Conflicts of interest}

There are no conflicts of interest.

\section{References}

1. Information about COVID-19, Coronavirus in India. Official Public Service Announcement on Coronavirus from the Government. Available from: www.mygov.in/covid-19. [Last cited on 2020 Jun 06].

2. Tan BS, Tay KH, Tan BH, Chung JW, Gangi A, Binkert C, et al. Joint CIRSE-APSCVIR checklist for preparing your IR service for COVID-19. Available from: www.cirse.org/education/ covid-19-resource-centre. [Last cited on 2020 Apr 15].

3. Stempniak M. 6 steps interventional radiology can take to prepare for the coronavirus. Radiology Business February 27, 2020 [Online]. Available from: https://www.radiologybusiness.com/ topics/leadership/interventional-radiology-prepare-coronavirus. [Last cited on 2020 Mar 30].

4. OEIS Procedural Conduct Recommendations pertaining to COVID-19. Available from: https://evtoday.com/news/ oeis-provides-interventional-procedure-recommendations-during -covid-19-crisis. [Last cited on 2020 Apr 15].

5. Mirza SK, Tragon TR, Fukui MB, Hartman MS, Hartman AL. Microbiology for radiologists: How to minimize infection transmission in the radiology department. Radiographics 2015;35:1231-44.

6. Modes of transmission of virus causing COVID-19: implications for IPC precaution recommendations. Available from: https://www.who.int/news-room/commentaries/ detail/modes-of-transmission-of-virus-causing -covid-19 -implications-for-ipc-precaution-recommendations. [Last cited on 2020 Apr 10].

7. Ortega R, Obanor O, Yu P. Putting on and removing personal protective equipment. N Eng J Med 2015;372:2464-5.

8. Use Personal Protective Equipment (PPE) When Caring for Patients with Confirmed or Suspected COVID-19. Available from: https:// www.cdc.gov/coronavirus/2019-ncov/downloads/A_FS_HCP_ COVID19_PPE.pdf. [Last cited on 2020 Apr 10].

9. Chandy PE, Nasir MU, Srinivasan S, Klass D, Nicolaou S, Babu SB. Interventional radiology and COVID-19: Evidence-based measures to limit transmission. Diagn Interv Radiol 2020;26:236-40.

10. International Standard ISO 14644- Cleanroom and associated controlled environments-Part 1-12. Available from: https://www. iso.org/obp/ui/\#!iso:std:69432:en. [Last cited on 2020 Apr 24].

11. National Guidelines for Infection Prevention and Control in Healthcare Facilities. Available from: https://www.mohfw.gov. in/pdf/National\%20Guidelines\%20for\%20IPC\%20in\%20HCF\%20 -\%20final\%281\%29.pdf. [Last cited on 2020 Apr 10].

12. ACEP Guideline on COVID-19: Ultrasound Machine and Transducer Cleaning. Available from: https://www. acep.org/globalassets/new-pdfs/guideline-on-covid-19ultrasound-machine-and-transducer-cleaning_policy_033120.pdf. [Last cited on 2020 Mar 31].

13. Environmental Protection Agency. List N: Disinfectants for Use Against SARS-CoV-2. Available from: https://www.epa.gov/ pesticide-registration/list-n-disinfectants-use-against-sars-cov-2. [Last cited on 2020 Apr 24].

14. Huang Z, Zhao S, Li Z, Chen W, Zhao L, Deng L, et al. The battle against coronavirus disease 2019 (COVID-19): Emergency management and infection control in a radiology department. J Am Coll Radiol 2020;17:710-6.

15. Enforcement Policy for Sterilizers, Disinfectant Devices, and Air Purifiers During the Coronavirus Disease 2019 (COVID-19) Public Health Emergency Guidance for Industry and Food and Drug Administration Staff. March 2020. Available from: https://www. fda.gov/media/136533/download. [Last cited on 2020 Apr 10].

16. Babu A, Padmanabhan A, Khera P, Sonwalkar H. Better safe than sorry: Interventional radiology should be prepared for the coronavirus disease 2019 pandemic. J Clin Interv Radiol ISVIR 2020;4:38-41.

17. Keshava SN, Gupta A, Pant R, Sutphin DP, Kalva S. Practice of interventional radiology during the COVID-19 pandemic. J Clin Interv Radiol ISVIR 2020;4:1-2. 